\title{
Efeitos da fenilbutazona na cicatrização de feridas cutâneas experimentais em equinos
}

\author{
Phenylbutazone effects on experimental wound healing in horses \\ Carlos Alberto HUSSNI ${ }^{1}$; Tanja Melanie GROH ${ }^{1}$; Ana Liz Garcia ALVES ${ }^{1}$; Adalberto José CROCCI ${ }^{2}$; \\ José Luiz de Mello NICOLETTI ${ }^{1}$; Marcos Jun WATANABE ${ }^{1}$ \\ ${ }^{1}$ Departamento de Cirurgia e Anestesiologia Veterinária da Faculdade de Medicina Veterinária e Zootecnia da Universidade \\ Estadual Paulista, Botucatu-SP \\ ${ }^{2}$ Departamento de Bioestatística do Instituto de Biociências da Universidade Estadual Paulista, Botucatu-SP
}

\begin{abstract}
Resumo
No processo de cicatrização por segunda intenção de feridas cutâneas experimentalmente induzidas em equinos, avaliaram-se os efeitos da fenilbutazona e comparou-se a cicatrização entre as regiões torácica e lombar. Utilizaram-se dez equinos, dos quais se retirou fragmentos circulares de pele de dois centímetros de diâmetro das regiões lombares e torácicas direita e esquerda. Os equinos foram distribuídos em dois grupos, sendo o primeiro controle, recebendo água destilada a cada 12 horas, durante cinco dias. O outro grupo foi tratado com fenilbutazona $(4,4 \mathrm{mg} / \mathrm{kg}) \mathrm{com}$ o mesmo intervalo e período do grupo controle. As feridas foram tratadas diariamente com Líquido de Dakin, momentos quando se procederam as observações macroscópicas. A cada 72 horas procederam-se as mensurações das feridas. Para análise histológica realizou-se biópsias no sexto e décimo quinto dia. O tempo total de reparo das feridas no grupo tratado foi maior em aproximadamente 12 dias (37 dias para o grupo controle e 49 dias para o grupo tratado). Não se observou diferença significativa do tempo de cicatrização entre as feridas torácicas e lombares de um mesmo grupo. As avaliações macroscópicas e histopatológicas mostraram o efeito inibidor da fenilbutazona quando comparada com o grupo controle na cicatrização de feridas cutâneas por segunda intenção em equinos.
\end{abstract}

Palavras-chave: Cicatrização. Ferida. Fenilbutazona. Equinos.

\begin{abstract}
The purpose of this study was to investigate phenylbutazone effects on second intention wound healing, and to compare the healing process between the thoracic and lumbar areas. Ten horses were submitted to circular full-thickness wound produced on both sides of the thoracic and lumbar areas. Animals were gathered into two experimental groups, one receiving daily IV injections of phenylbutazone $(4,4 \mathrm{mg} / \mathrm{kg}$ ) and the other (control group) distillated water for five days. All wounds were daily treated with local Dakin's solution. The wound contraction rates were determined by serial measurements each 72 hours. At the 6th and 15th post surgical days, biopsies were performed for histological analysis. Thoracic and lumbar wound contraction was decreased in the phenylbutazone group. The time to complete healing was significantly greater in phenylbutazone group (49 days) than in control group (37 days). There was no significant difference between thoracic and lumbar area in the same group. Gross and histopathology analysis showed the inhibitory effect of phenylbutazone on the second-intention wound healing when compared to the control group.
\end{abstract}

Keywords: Healing. Wound. Phenylbutazone. Horses.

\section{Introdução}

As feridas cutâneas em equinos possuem alta ocorrência resultando em perdas funcionais e econômicas na espécie, o que determina a busca da reparação com a recuperação das características funcionais e cosméticas da pele $e^{1,2,3,4,5}$.

No processo de regeneração, o tecido neoformado é semelhante ao pré-existente, enquanto que a cicatrização compõe novo tecido com arranjo que difere do original ${ }^{6}$. Neste processo, após a lesão cutânea ocorre síntese de tecido conectivo denso, contração e epitelização $0^{4,7}$ e a cicatrização pode ser classificada por

\section{Correspondência para:}

Carlos Alberto Hussni

DCAV - FMVZ - UNESP Rubião Jr - Botucatu - SP CEP 18618-000

Tel 14-38116252 ramal 23 Fax 14-38116072

e-mail: cahussni@fmvz.unesp.br

Recebido: 30/07/2008

Aprovado: 01/04/2010

Agradecimentos: Conselho Nacional de Desenvolvimento Científico e Tecnológico - CNPq - PIBIC 
primeira, segunda ou ainda por terceira intenção $0^{2,8,9,10}$, com a cicatrização por segunda intenção sendo a única opção em grande número de casos.

Devido à complexidade do processo cicatricial cutâneo, quatro fases são descritas na literatura: inflamatória, debridamento, reparativa e maturação ${ }^{1,3,5,7}$. A fase inflamatória inicia-se imediatamente após a ocorrência da lesão, em resposta à liberação de mediadores químicos ${ }^{10,11}$. Seis horas após a ocorrência da lesão, inicia-se a fase de debridamento, caracterizando-se pela presença de neutrófilos e predominantemente macrófagos $^{3,5}$. A fase de reparação envolve epitelização da superfície da ferida, migração dos fibroblastos necessários para a formação de colágeno, formação de tecido de granulação e contração da ferida, que consiste na redução da ferida pelo movimento centrípeto da pele ${ }^{3,5}$. Na fase de maturação, as fibras de colágeno são modificadas e tornam-se mais compactas e densas, o número de fibroblastos decresce e as fibras desenvolvem a orientação definitiva. A cicatriz torna-se mais plana, pálida e discretamente amolecida, podendo durar de meses a anos para que ocorra a maturação total $^{5,12}$.

A fenilbutazona é um anti-inflamatório não esteróide (AINE) empregado no tratamento de inflamações em diferentes tecidos ${ }^{13}$, que age inibindo a síntese de prostanóides derivados do ácido araquidônico, por meio da enzima ciclooxigenase, diminuindo assim, os sinais da inflamação ${ }^{14}$. Em comparação à terapia com corticosteroides, onde ao mesmo tempo em que exercem um efeito anti-inflamatório retardam a fibroplasia, os AINEs suprimem a síntese de prostaglandinas, aliviam a dor e reduzem a vasodilatação sem, aparentemente, apresentarem efeitos sobre a fibroplasia ${ }^{15}$. Porém, podem reduzir a migração leucocitária "in vitro" ${ }^{16} \mathrm{e}$ interferir na qualidade da cicatrização $0^{3,5,13}$. Considerada a ampla utilização da fenilbutazona no tratamento das diversas enfermidades em equinos, especialmente nos casos em que há solução de continuidade da pele, este trabalho objetivou observar os possíveis efeitos da fe- nilbutazona na cicatrização de pele por segunda intenção e verificar possíveis diferenças de cicatrização entre as regiões lombar e torácica de equinos adultos.

\section{Material e Método}

O presente experimento foi aprovado pela câmara de ética em experimentação animal - CEEA/FMVZUNESP e registrada sob $\mathrm{n}^{\circ} 12 / 08$.

Foram utilizados 10 equinos adultos, machos castrados, sem raça definida e clinicamente sadios. Após preparação de rotina, cada animal foi contido em tronco e tranquilizado com $0,12 \mathrm{mg} / \mathrm{kg}$ de romifidina (Sedivet - Boehringer Ingelheim do Brasil Química e Farmacêutica Ltda - São Paulo, SP), pela via intravenosa. Os locais das feridas foram estabelecidos em: uma no hemitórax direito e outra no esquerdo, uma na região lombar direita e outra na esquerda, totalizando quatro feridas por animal. Procedeu-se a tricotomia e antissepsia com álcool iodado a 5\%. As feridas foram produzidas com um punch de dois centímetros de diâmetro, removendo-se fragmentos circulares de pele.

Os animais foram distribuídos aleatoriamente em dois grupos de cinco, sendo que os equinos do grupo controle (grupo C) receberam água bidestilada (Ariston Industria Química e Farmacêutica Ltda - São Paulo, SP) no volume de 2,2 mL/100 kg pela via intravenosa, a cada 12 horas durante cinco dias, completando um total de 10 aplicações. Os equinos do grupo fenilbutazona (grupo F), pela mesma via de administração, intervalo e número de aplicações, receberam a fenilbutazona (Butazolidina, Novartis Biociências S.A.- São Paulo, SP) na dose de $4,4 \mathrm{mg} / \mathrm{kg}$. Até o final do experimento todas as feridas foram tratadas com a aplicação tópica diária de Líquido de Dakin (Indústria Farmacêutica Rioquímica Ltda - São José do Rio Preto, SP) diluído a 0,005\%.

A avaliação macroscópica da cicatrização foi realizada nas feridas torácicas e lombares do lado esquerdo, consistindo de exames a cada 72 h quanto à pre- 
sença de crostas e exsudação, e imediatamente após o procedimento cirúrgico, a cada $72 \mathrm{~h}$ e até o fechamento total, as bordas das feridas foram demarcadas em filmes plásticos transparentes com caneta marcadora de retro projetor. Posteriormente, estas medidas foram submetidas à análise de planimetria com auxílio de um planímetro (Planimeter KP 23 - Koizumi, Japão), obtendo-se assim, a área das feridas até $0,25 \mathrm{~cm}^{2}$ como limite mínimo de mensuração do planímetro.

As feridas torácicas e lombares do lado direito foram destinadas a biópsias realizadas com punch de dois milímetros de diâmetro no sexto e no décimo quinto dia pós-cirúrgico. $\mathrm{O}$ material foi fixado em formaldeído tamponado a $10 \%$, processado e corado com Hematoxilina e Eosina e Tricrômio de Masson, sendo os parâmetros avaliados: integridade do epitélio, presença de edema na derme, organização de tecido conjuntivo frouxo e denso, presença de hemorragia, neoformação vascular e os tipos celulares nas fases do processo.

A análise estatística baseou-se em duas situações: a comparação entre os grupos e entre os locais das feridas, pela média de dias decorridos até a cicatrização total, através da Análise de Perfil ${ }^{17}$, com transformação x. Comparação entre os grupos e entre os locais das V̌ridas, pela diminuição média diária da área da ferida, obtida pelo coeficiente de regressão linear da área em função dos dias de observação.

\section{Resultados e Discussão}

Até o nono dia após a indução das feridas, estas apresentaram aumento de volume nas bordas devido a edema perilesional em forma de halo de aproximadamente dois centímetros de largura. No grupo F o halo foi de aproximadamente um centímetro de largura, desaparecendo até o sexto dia pós-operatório. Esse aumento de volume é a expressão macroscópica do edema inflamatório ${ }^{5,11}$. A histopatologia ao sexto dia comprovou esses resultados, uma vez que a bi- ópsia correspondente evidenciou presença de edema discreto no grupo $\mathrm{C}$ e ausência do mesmo no grupo F. Esses resultados se justificam, pois a fenilbutazona inibe a síntese de prostaglandinas diminuindo, assim, a inflamação ${ }^{14}$.

No grupo C houve presença de secreção sero-fibrinosa, chegando, por vezes, a fibrino-purulenta, com crostas espessas, vermelho escuras, opacas e facilmente removíveis do segundo ao $12^{\circ}$ dia pós-operatório. Após esta fase, as mesmas feridas mostraram-se secas, passando a apresentar crostas finas, amareladas, translúcidas e removíveis com dificuldade. Nos animais do grupo F, do segundo dia até o final do experimento, as feridas permaneceram secas, sem exsudações, com crostas finas, amareladas, translúcidas e não removíveis. Este resultado sugere que a fenilbutazona diminui a formação de exsudato ${ }^{18}$. As feridas do grupo $\mathrm{C}$ a partir do $12^{\circ}$ dia foram semelhantes às feridas do grupo $\mathrm{F}$ caracterizadas desde o início do processo, mantendo este aspecto até a cicatrização total. As crostas são formadas pela dessecação da superfície da ferida sendo compostas por sangue coagulado, fibrina, colágeno ressecado e debris celulares ${ }^{19}$. Feridas mais exsudativas tendem a formar crostas mais espessas e mais facilmente removíveis. Isto confere menor proteção à ferida e, portanto, é preferível a presença de menor exsudação, seca e com crosta protetora aderida. Nas feridas do grupo $\mathrm{C}$, até o $12^{\circ}$ dia, as crostas mostraram-se mais espessas com maior presença de exsudato. Crostas mais finas, com menos exsudato ocorreu em todo o período de observação nas feridas no grupo $\mathrm{F}$ e a partir do $12^{\circ}$ dia pós-operatório no grupo C.

Nos grupos C e F o tecido de granulação esteve presente em todas as feridas a partir do sexto dia pósoperatório, permanecendo até o $18^{\circ}$ dia no grupo $\mathrm{C}$ e até o $24^{\circ}$ dia no grupo F. A partir desses dias o fundo apresentou-se amarelado e liso. A granulação observada nas feridas de ambos os grupos mostrou inicialmente a presença de grânulos grandes e ver- 
melho brilhantes evoluindo para grânulos pequenos e rosados, conforme descrito ${ }^{11,18}$. Aspectos das feridas estão apresentados na figura 1.
A planimetria das feridas lombares de ambos os grupos mostrou aumento da área da ferida no terceiro dia pós-operatório e diminuição no sexto dia, tornando-se inferior à área inicial, diminuindo gra-

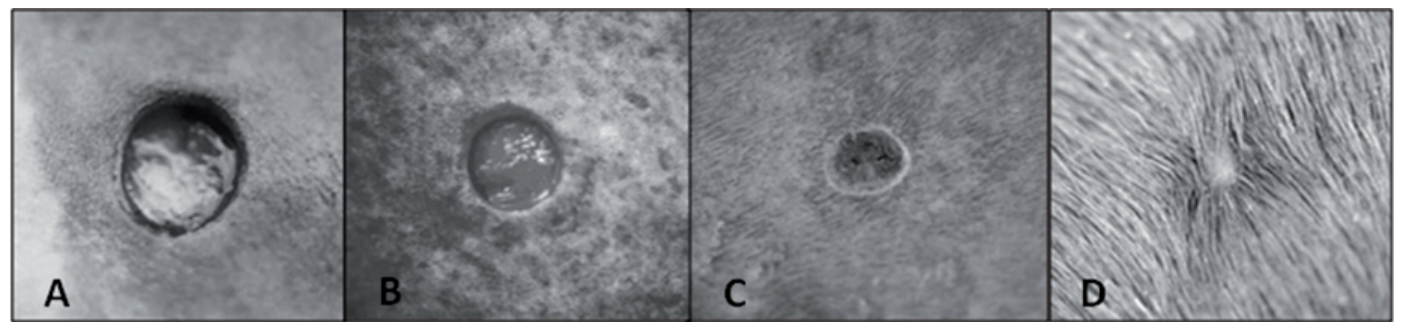

Figura 1 - Aspectos das feridas torácicas de animais do grupo controle. A - Imediatamente após a indução, com coágulo sanguíneo no bordo da ferida. B - No terceiro dia observável o edema perilesional. C - No 15 dia, com crosta aderida e delgada. D - Cicatrização completa da ferida

dativamente, até a cicatrização total. Nas feridas torácicas não se observou esse aumento no terceiro dia pós-operatório e sim uma discreta redução da área da superfície. A partir do sexto dia as feridas nas regiões torácicas, de ambos os grupos, comportaramse de forma semelhante às feridas nas regiões lombares. Em cada local da ferida os grupos C e F diferiram estatisticamente $(\mathrm{p}<0,05)$ quanto à diminuição da área da ferida (Tabela 1)

Tabela 1 - Retração média diária da área da superfície das feridas $\left(\mathrm{cm}^{2}\right)$ torácica e lombar para os grupos controle (C) e fenilbutazona (F) com os resultados da análise estatística, comparandose as feridas entre os locais (torácica e lombar) e entre os grupos (C e F) - Botucatu, SP - 2008

\begin{tabular}{ccc}
\hline Grupo & Ferida Torácica & Ferida Lombar \\
\hline C & $0,25 \mathrm{~b}$ & $0,21 \mathrm{~b}$ \\
F & $0,16 \mathrm{a}$ & $0,15 \mathrm{a}$ \\
\hline
\end{tabular}

Letras iguais os resultados não diferem. $\mathrm{a}<\mathrm{b} ; \mathrm{p}<0,05$

O tempo médio da cicatrização das feridas no grupo $\mathrm{C}$ foi de 37 dias, sendo a média para as feridas torácicas 37,8 dias e para as lombares 37,2 (Figura 2). Para cada grupo (controle e fenilbutazona), a diferença en- tre as feridas torácicas e lombares não foi significativa conforme descrito ${ }^{20,21}$ (Tabela 2). No grupo F o tempo médio de cicatrização foi de 49 dias, consideradas as médias das feridas torácicas em 48,6 dias e as médias das lombares 49,8 dias (Figura 2). A diferença obtida entre o tempo de cicatrização entre as duas regiões não foi significativa (Tabela 2). Comparando-se o tempo total de cicatrização das feridas torácicas e lombares entre os grupos $\mathrm{C}$ e F, observou-se diferença significativa ( $\mathrm{p}=0,051)$ (Tabela 2$)$. O tempo total de cicatrização para as regiões estudadas e entre os grupos tiveram a média acima do descrito em experimento realizado em condições semelhantes ${ }^{5}$, com maior tempo evidente para as feridas do grupo tratado com fenilbutazona.

Tabela 2 - Tempo médio total de cicatrização (dias) e resultados estatísticos da Análise de Perfil com transformação em $\sqrt{\mathrm{x}}$ e a média original do tempo total de cicatrização (dias). Comparamse os grupos (C e F) e os locais das feridas (torácica e lombar) - Botucatu, SP - 2008

\begin{tabular}{crr}
\hline Grupo & Ferida Torácica & Ferida Lombar \\
\hline C & $6,1(37,8) \mathrm{b}$ & $6,1(37,2) \mathrm{b}$ \\
$\mathrm{F}$ & $6,9(48,6) \mathrm{a}$ & $7,0(49,8) \mathrm{a}$ \\
\hline
\end{tabular}

Letras iguais os resultados não diferem. $a>b ; p=0,051$ 


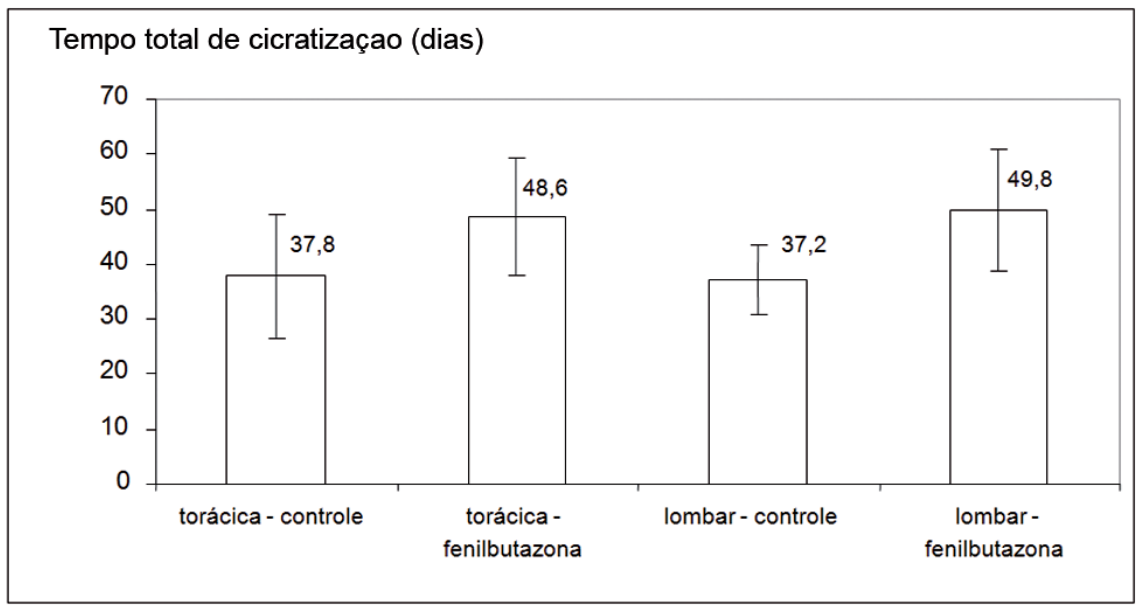

Figura 2 - Médias com desvio padrão do tempo total de cicatrização (dias) das feridas nas regiões lombar e torácica para os tratamentos controle (grupo C) e com fenilbutazona (grupo F)

Os exames histopatológicos ao sexto dia pós-operatório mostraram presença de edema discreto no grupo $\mathrm{C}$ e ausência do mesmo no grupo $\mathrm{F}$, semelhante ao descrito ${ }^{14,16,22}$, com neovascularização evidente em ambos os grupos. A neovascularização foi mais acentuada nas feridas do grupo F. A presença de fibroblastos foi moderada, com ausência de deposição de colágeno e de fibra conjuntiva no grupo $\mathrm{C}$, sendo que para o grupo $\mathrm{F}$ a presença de fibroblastos foi acentuada. Em ambos os grupos houve presença acentuada de eosinófilos e mononucleares, características do exsudato inflamatório ${ }^{10}$. O grupo $\mathrm{C}$ apresentou presença variável de neutrófilos, estes ausentes nas feridas do grupo F. Nos animais do grupo C houve início de epitelização das feridas, estando a epitelização ausente nas feridas grupo F, semelhante ao descrito ${ }^{5}$.

A histopatologia realizada no $15^{\circ}$ dia pós-operatório mostrou neoformação vascular acentuada em ambos os grupos. No grupo C observou-se presença moderada de fibroblastos, deposição discreta a moderada de colágeno e presença esporádica de fibra conjuntiva, eventos estes descritos ${ }^{23}$. Nas feridas do grupo $\mathrm{F}$ houve ausência de fibroblastos, de deposição colágeno e presença acentuada de fibra conjuntiva diferindo do grupo $\mathrm{C}$ que sugere o aparecimento tardio da fibra conjuntiva. Nos equinos do grupo $\mathrm{C}$ houve predomínio da regeneração epitelial, parcial ou total. Nos animais do grupo F, observou-se regeneração parcial do epitélio em cerca da metade das feridas e ausência do mesmo nas demais, caracterizando epitelização mais tardia quando comparadas com as feridas do grupo C, semelhante ao exposto ${ }^{5}$. Quanto às características macroscópicas e histopatológicas discutidas não se observou diferenças entre as feridas das regiões torácica e lombar, mas sim entre os grupos C e F.

\section{Conclusões}

A fenilbutazona retarda a cicatrização de pele por segunda intenção em equinos. Não existem diferenças na cicatrização por segunda intenção entre lesões cutâneas nas regiões torácicas e lombares. 


\section{Referências}

1. BERTONE, A. L. Principles of wound healing. Veterinary Clinics North America: Equine Practice, v. 5, n. 3, p. 449-463, 1989.

2. WOUK, A. F. P. F.; MICHELOTTO JR., P. V. Relato preliminar do uso de um novo curativo biológico (Biofill) em feridas de pele com grande perda de substância em um equino. Hora Veterinária, v. 8, p. 31-34, 1989.

3. STASHAK, T. S. Equine wound management. Philadelphia: Lea \& Febiger, 1991. 278 p.

4. SCHUMACHER, J.; BRUMBAGH, G. W.; HONNAS, C. M.; TARLEY, R. J. Kinetics of healing of grafted and nongrafted wounds on the distal portion of the forelimbs of horses. American Journal Veterinary Research, v. 53, n. 9, p. 1568$1571,1992$.

5. HUSSNI, C. A.; GIANINI, C. G.; ALVES, A. L. G.; NICOLETTI, J. L. M.; THOMASSIAN, A.; CROCCI, A. J.; SEQUEIRA, J. L. Cicatrização cutânea por segunda intenção em equinos tratados com vedaprofeno. Archives of Veterinary Science, v. 9, n. 1, p.87-97, 2004.

6. SILVER, I. A. The mecanics of wound healing. Equine Veterinary Journal, v. 11, n. 2, p. 93-96, 1979.

7. JOHNSTON, D. E. The process in wound healing. Journal American Animal Hospital Association, v. 13, p. 186-196, 1977.

8. TURNER, A. S.; MCILWRAITH, C. W. Técnica cirúrgica em animais de grande porte. São Paulo: Roca, 1985. 341 p.

9. AUER, J. A.; STICK, J. A. Equine surgery. 2. ed. Philadelphia: Saunders, 1999. $937 \mathrm{p}$.

10.BANKS, W. J. Histologia veterinária aplicada. 2. ed. São Paulo: Manole, 1992. 629 p.

11.BARRETO-NETTO, M.; MONTENEGRO, M. F.; BRITO, T. de; ADNRADE, Z. A. Patologia: processos gerais. 2. ed. Niterói: Programa Interuniversitário de Patologia, 1984. 188 p.

12.BOJRAB, M. J. Wound management. Modern Veterinary Practice, v. 63, p. 791-796, 1982.
13. MCILWRAITH, C. W.; TROTTER, G. W. Joint disease in the horse. Philadelphia: Saunders, 1996. 490 p.

14. KALLINGS, P. Non-steroidal anti-inflammatory drugs. Veterinary Clinics North America: Equine Practice, v. 9, p. 523-541, 1993.

15.MAY, S. A.; LEES, P.; HIGGINS, A. J.; SEDEWICK, A. D. Inflammation: a clinical perspective. Veterinary Records, v. 120 , n. 120 , p. $514-517,1987$.

16.DAWSON, J.; LEES, P.; SEDGWICK, A. D. Action of nonsteroidal anti-inflammatory drugs on equine leucocyte movement in vitro. Journal Veterinary Pharmacology Therapeutics, v. 10, p. 150-159, 1987.

17. MORRISON, D. F. Multivariate Statistical Analysis. 3. ed. New York: Mc Graw-Hill, 1990. 415 p.

18.COTRAN, R. S.; KUMAR, V.; ROBBINS, S. L. Robbins patologia estrutural e funcional. 4. ed. Rio de Janeiro: Guanabara Koogan, 1991. p. 33-72.

19.FITCH, R. B.; SWAIM, S. T. The role of epithelialization in wound healing. Small Animal Wound Healing, v. 17, n. 2, p. 167-176, 1995.

20.JACOBS, K. A.; LEACH, D. H.; FRETZ, P. B.; TOWNSEND, H. G. G. Comparative aspects of healing of excisional wounds on the leg and body of horses. Veterinary Surgery, v. 13, n. 2, p. 83-90, 1984.

21. KNOTTENBELT, D. C. Equine wound management: are there significant differences in healing at different sites on the body? Journal Veterinary Dermatology, v. 8, p. 273-290, 1997.

22.LEES, P.; HIGGINS, A. J. Clinical pharmacology and therapeotic uses of non-steroidal anti-inflammatory drugs in the horse. Equine Veterinary Journal, v. 17, p. 83-96, 1985.

23. ORGILL, D.; DEMLING, R. H. Current concepts and approaches to wound healing. Critical Care Medicine, v. 16, n. 9, p. 899-907, 1988. 